\title{
Successful Treatment of Recurrent Triple-Negative Breast Cancer with Combination of Targeted Therapies
}

\author{
Stanislaw R. Burzynski, Alejandro Marquis, Eva Nagy-Kubove, Tomasz Janicki \\ Burzynski Clinic, 9432 Katy Freeway, Houston, USA. \\ E-mail: srb@burzynskiclinic.com
}

Received March 5 ${ }^{\text {th }}$, 2011; revised April 26 ${ }^{\text {th }}$, 2011; accepted May 7 $7^{\text {th }} 2011$.

\begin{abstract}
We present an interesting case of a 56-year-old female diagnosed with invasive high-grade triple-negative breast cancer, who developed diffuse liver metastases following lumpectomy and combination chemotherapy with docetaxel, doxorubicin and cyclophosphamide, re-excision and radiation therapy. Restaging CT and PET scans revealed massive involvement of the liver. She was treated with a combination of gene targeted and cytotoxic chemotherapy including capecitabine, erlotinib, bevacizumab and phenylbutyrate. She tested weakly positive for HER-2 despite prior negative FISH, which prompted us to add trastuzumab to her regimen. Baseline CT revealed five liver tumors-the sum of the products of the two largest perpendicular diameters was $110 \mathrm{~cm}^{2}$. Follow-up CT after three months of treatment revealed $62 \%$ decrease in total tumor load. More than $50 \%$ decrease in tumor size persisted on two follow-up CT scans, confirming partial response. She developed progressive disease after 15 months of treatment. A group of 16 women, including this patient, diagnosed with triple negative breast cancer with distant metastases were treated by our team with a combination of gene targeted therapy and chemotherapy. Six percent of patients obtained partial response, 25\% minor response, 31\% stable disease, and 38\% progressive disease. The median duration of treatment in patients who relapsed after the second-, third- and fourth- to seventh-lines of chemotherapy was 59 weeks, 22 weeks and 17 weeks, respectively. Comparison of results obtained with cytotoxic chemotherapy revealed that MDT in the second-and third-lines was only nine and four weeks, respectively. In conclusion, this case report indicates that it is possible to obtain durable objective response of recurrent TNBC with a combination of gene targeted agents.
\end{abstract}

Keywords: Breast Cancer, Cancer Treatment, Targeted Therapy, Triple-Negative Breast Cancer

\section{Introduction}

Gene expression studies have identified multiple subtypes of breast cancer with different "genetic signatures" $[1,2]$. Triple-negative breast cancer (TNBC) which does not over-express HER-2 (human epidermal growth factor receptor 2), estrogen receptor, or progesterone receptor creates the biggest challenge in the treatment of metastatic breast disease [3]. Patients diagnosed with TNBC have shown an inferior prognosis with the increased likelihood of distant recurrence including brain metastases within five years of diagnosis. The mean time to distant metastases with TNBC is approximately 50\% shorter than that of other breast cancer groups [1]. The mean time to death for patients diagnosed with TNBC is 4.2 years compared to 6 years for other groups [1]. Due to the absence of specific genetic markers, gene-targeted therapy is not often used for TNBC compared to other cancers. Traditional cytotoxic chemotherapy remains the standard treatment. Patients with TNBC relapse quickly on chemotherapy, and, as a result the median duration of treatment (MDT) was proposed as a surrogate for duration of treatment response [4,5]. The feedback up-regulation of compensatory signaling networks has been observed with single gene targeted agents that were not successful in the treatment of TNBC. It has been proposed that successful treatment of this type of cancer requires combined inhibition of PI3K/AKT and RAS/ RAF/MEK signaling pathways [6]. The studies on HER-2/neu, EGFR, and VEGF in serum indicate that such tests can be used to select additional targeted agents which effect single genes [7-9].

Here we report the successful treatment of metastatic TNBC with combination targeted therapy, and we dis- 
cuss MDT for a group of 16 women including this patient, whose treatment was based on the same principle.

\section{Case Report}

In January 2007 a 56-year-old Lebanese female was diagnosed with invasive high-grade TNBC with regional lymph node metastases. Her initial treatment included lumpectomy, TAC chemotherapy (six cycles of docetaxel, doxorubicin, and cyclophosphamide), reexcision, and radiation therapy, through September 2, 2007, which resulted in complete response. In January 2008, she developed diffuse liver metastases, and presented to our clinic on February 11, 2008, complaining of abdominal pain, decreased energy level, nausea, night sweats and depression (Figure 1).

Baseline PET/CT (positron emission tomography/ computed tomography) revealed multiple hypermetabolic lesions replacing most of the normal liver structure as

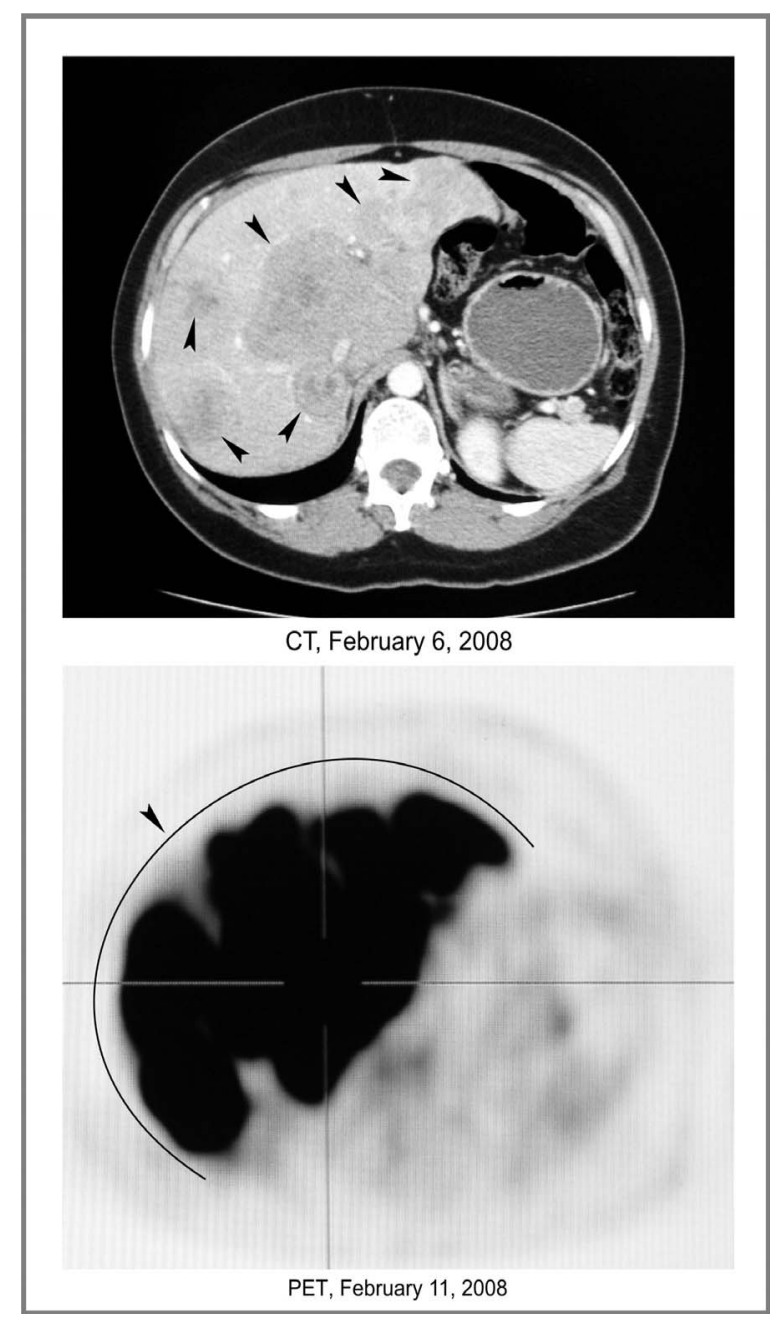

Figure 1. Baseline PET/CT. Multiple liver tumors. shown in Figure 1. She signed the informed consent and began treatment with capecitabine $1650 \mathrm{mg}$ po daily, erlotinib $150 \mathrm{mg}$ po daily, bevacizumab $10 \mathrm{mg} / \mathrm{kg} \mathrm{IV}$ every 2 weeks, and phenylbutyrate $6 \mathrm{~g}$ po daily. She tested weakly positive for HER-2 despite prior negative FISH (fluorescent in situ hybridization); and she was started on trastuzumab.

Baseline CT of February 6, 2008, revealed five tumors; the sum of the products of the two largest perpendicular diameters was $110 \mathrm{~cm}^{2}$. Follow-up CT revealed decreased tumor size and by May 8, 2008 PR was reached (62\% decrease of the total tumor load) (Figure 2). Figure 2 represents a CT of May 8, 2008, which documents a marked decrease of the lesions.

More than 50\% decrease persisted on CT scans of July 7 and September 26, 2008. The PET/CT (Figure 3) confirms the patient's partial response (PR). Her last CT of March 16, 2009, showed 49\% decrease. Her radiological improvement coincided with disappearance of symptoms, reduction of tumor markers, and normalization of liver tests. Baseline CEA, CA 27-29, and CA 125 were correspondingly 1502 ng/mL, $632 \mathrm{U} / \mathrm{mL}$, and $386 \mathrm{U} / \mathrm{mL}$. By May 5 2008, they had decreased to $339 \mathrm{ng} / \mathrm{mL}, 115$ $\mathrm{U} / \mathrm{mL}$, and $54 \mathrm{U} / \mathrm{mL}$.

On June 23, 2008, her tumor markers began to climb. Capecitabine and erlotinib were discontinued, and replaced by lapatinib $750 \mathrm{mg}$ po daily, sorafenib $200 \mathrm{mg}$ po bid, and vorinostat $100 \mathrm{mg}$ po daily. Follow-up CT of September 26, 2008, confirmed stabilization of her disease. Due to poor tolerance, lapatinib and bevacizumab were discontinued on January 7, 2009. Due to increased tumor markers, vinorelbine $20 \mathrm{mg} / \mathrm{m}^{2} \mathrm{IV}$ Days 1 and 15 was added to the treatment and was continued until April 7, 2009. CT of March 16, 2009 showed 49\% decrease compared to baseline. In May 2009 her tumor markers

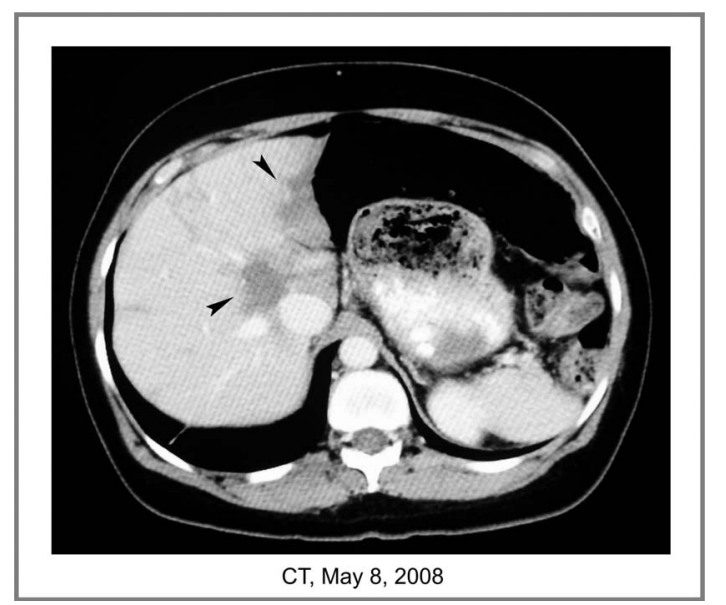

Figure 2. Beginning of partial response. Tumor size decreased by $62 \%$. 


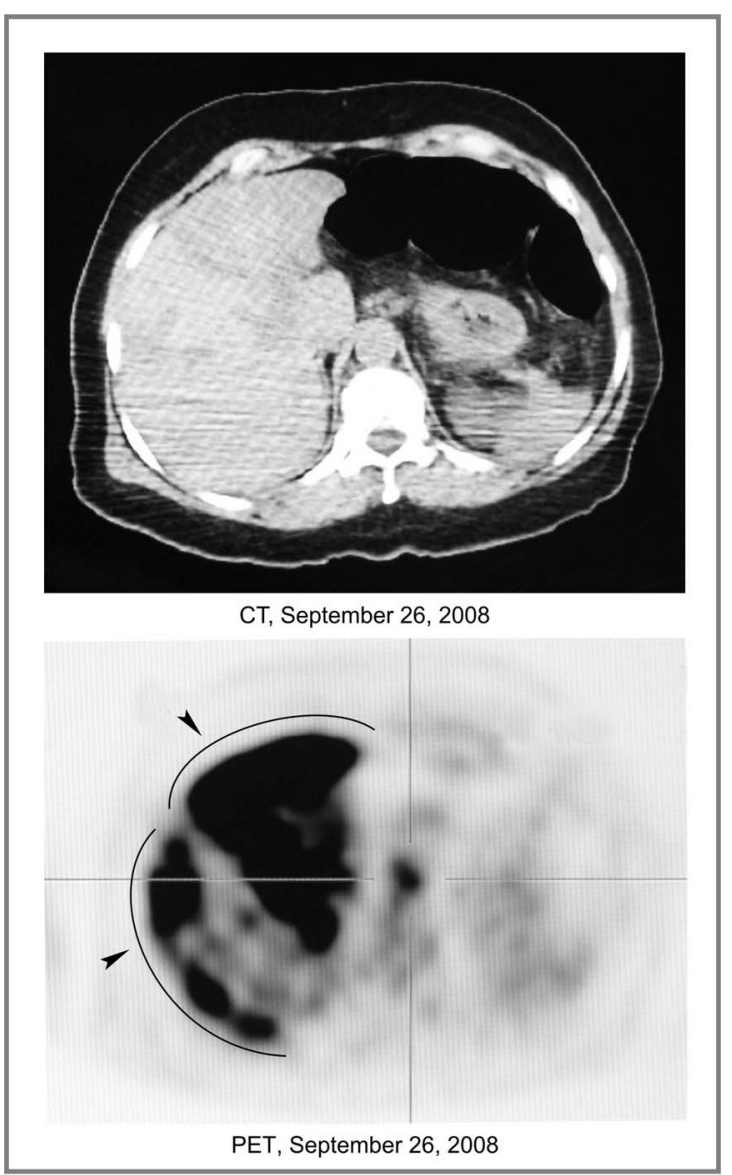

Figure 3. Continuation of partial response. Tumor size decreased by $50.3 \%$.

continued to rise. CEA increased to $1912 \mathrm{ng} / \mathrm{mL}$, and CA 27 - 29 to $1007 \mathrm{U} / \mathrm{mL}$ (May 18, 2009).

The treatment was discontinued on June 1, 2009, and the patient passed away on July 4, 2009.

\section{Discussion}

This patient failed combination chemotherapy with both anthracyclines and taxanes. The logical choice was the treatment with capecitabine, which is FDA-approved for such condition and recommended for TNBC [10]. Baseline elevation in serum concentrations of VEGF and HER-2/neu prompted us to add bevacizumab and trastuzumab [7-9].

Phenylbutyrate was added to inhibit AKT and ERK. Erlotinib was added after elevation of EGFR in serum. Sorafenib was introduced as VEGF and RAF inhibitor, and vorinostat was added for its synergistic effect with sorafenib, and inhibition of BCL-2 and BCL- $\mathrm{X}_{\mathrm{L}}$ (Figure 4) $[11,12]$. The diagram presented on Figure 4 provides a graphic explanation of the rationale for the treatment of this patient. The inhibition of two major signal transduction pathways, PI3K/AKT and RAS/RAF/MET, plays a crucial role in securing the response in TNBC. Cancer growth stimulatory signals are represented by red arrows and inhibitory interventions by green arrows and break symbols. Likewise cancer growth stimulatory proteins are shown in red and yellow, growth factor receptors in yellow and oncogene products in orange. Apoptosis inducing proteins are shown in green. It is postulated that the successful blockade of PI3K/AKT and RAS/RAF/MET pathways led to objective response in this patient.

A group of 16 women, including this case, diagnosed with TNBC with distant metastases, were treated by our team with a combination of gene-targeted therapies and cytotoxic chemotherapy. Prior treatments included surgery in $81 \%$ of patients, radiation therapy in $69 \%$, first-line chemotherapy in $37.5 \%$, second-line in $25 \%$, and the third- to sixth-line chemotherapy in $37.5 \%$. The selection of medications was based on determination of expression of EGFR, HER-2/neu, and VEGF oncogenes. Combination therapy included a tyrosine kinase and HDAC inhibitors and/or monoclonal antibodies. In patients who responded to chemotherapy (62\%. of patients), capecitabine, 5-florouracil, methotrexate, cyclophosphamide, vinorelbine, and/or temozolomide were added to their regimen. Six percent of patients obtained PR, 25\% minor response, 31\% stable disease (SD), and $38 \%$ progressive disease (PD). The MDT in the second-, third-, and fourth- to seventh-lines was 59 weeks, 22 weeks, and 17 weeks, respectively. Comparison of results obtained with cytotoxic chemotherapy reveals that MDT in the second- and third-line was only 9 and 4 weeks, respectively [5]. The treatment was tolerated very well, and in the majority of cases resulted in symptomatic improvement. In the majority of patients who did not develop PD, successful treatment consisted of simultaneous decrease of signaling along PI3K/AKT, RAS/RAF/MET and apoptosis pathways.

\section{Conclusions}

This case report indicates that it is possible to obtain an objective response in the treatment of recurrent TNBC with a combination of gene-targeted agents. The best results can be obtained with a combination of drugs that inhibit PI3K/AKT and RAS/RAF/MEK pathways. The selection of medication based on the determination of expression of the most important oncogenes was successful in accomplishing the disease control rate of $62 \%$ $(\mathrm{PR}+$ minor response $+\mathrm{SD})$ in a group of 16 women diagnosed with TNBC with distant metastases. The MDT in this group was substantially longer in comparison with MDT reported for cytotoxic chemotherapy. These findings may have implications for the design of clinical trials involving molecular profiling of individual patients with 


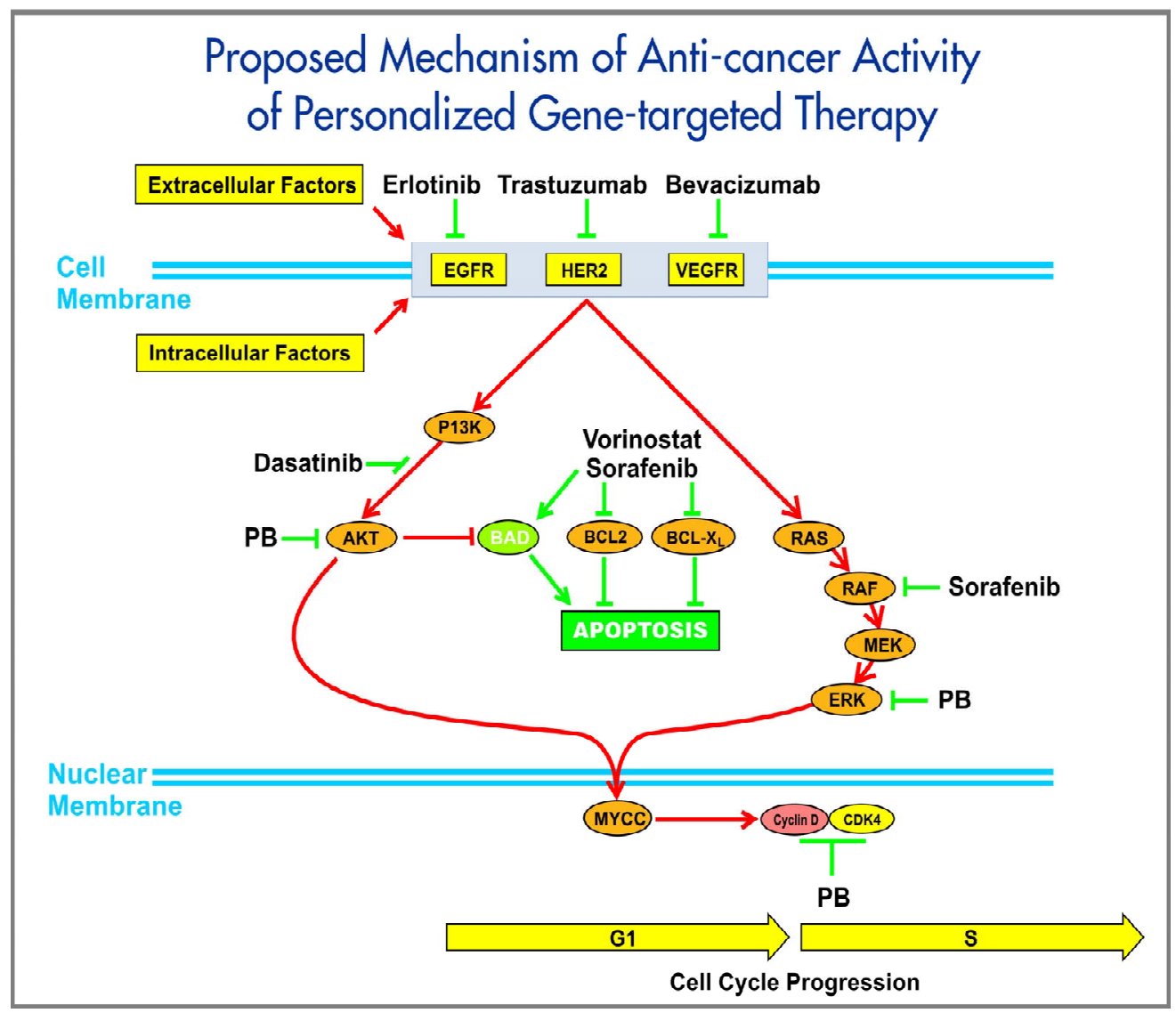

Figure 4. Successful treatment of triple-negative breast cancer requires combined inhibition of PI3K/AKT, RAS/RAF/MEK and apoptosis signaling pathways (PB-phenylbutyrate).

advanced TNBC.

\section{REFERENCES}

[1] R. Dent, M. Trudeau, K. I. Pritchard, W. M. Hanna, H. K. Kahn, C. A. Sawka, L. A. Lickley, E. Rawlinson, P. Sun and S. A. Narod, "Triple-Negative Breast Cancer: Clinical Features and Patterns of Recurrence," Clinical Cancer Research, Vol. 13, No. 15, 2007, pp. 4429-4434. doi:10.1158/1078-0432.CCR-06-3045

[2] C. A. Livasy, “Triple-Negative Breast Carcinoma,” Surgical Pathology, Vol. 2, No. 2, 2009, pp. 247-261.

[3] B. P. Schneider, E. P. Winer, W. D. Foulkes, J. Garber, C. M. Perou, A. Richardson, G. W. Sledge and L. A. Carey, "Triple-Negative Breast Cancer: Risk Factors to Potential Targets," Clinical Cancer Research, Vol. 14, No. 24, 2008, pp. 8010-8018. doi:10.1158/1078-0432.CCR-08-1208

[4] S. Verma, N. S. Wong, M. Trudeau, A. Joy, J. Mackey, G. Dranitsaris and M. Clemons, "Survival Differences Observed in Metastatic Breast Cancer Patients Treated with Capecitabine When Compared with Vinorelbine after Pretreatment with Anthracycline and Taxane," American Journal of Clinical Oncology, Vol. 30, No. 3, 2007, pp. 297-302. doi:10.1097/01.coc.0000258125.97090.3f
[5] F. Kassam, K. Enright, R. Dent, G. Dranitsaris, J. Myers, C. Flynn, M. Fralick, R. Kumar and M. Clemons, "Survival Outcomes for Patients with Metastatic TripleNegative Breast Cancer: Implications for Clinical Practice and Trial Design,” Clinical Breast Cancer, Vol. 9, No. 1, 2009, pp. 29-33. doi:10.3816/CBC.2009.n.005

[6] B. N. Rexer, R. Ghosh and C. L. Arteaga, "Inhibition of PI3K and MEK: It Is All about Combinations and Biomarkers," Clinical Cancer Research, Vol. 15, No. 14, 2009, pp. 4518-4520. doi:10.1158/1078-0432.CCR-09-0872

[7] J. S. Ross, J. A. Fletcher, G. P. Linette, J. Stec, E. Clark, M. Ayers, W. F. Symmans, L. Pusztai and K. J. Bloom, "The Her-2/neu Gene and Protein in Breast Cancer 2003: Biomarker and Target of Therapy,” Oncologist, Vol. 8, No. 4, 2003, pp. 307-325. doi:10.1634/theoncologist.8-4-307

[8] A. K. Swayampakula, V. Gadiyaram and J. Abraham, "Serum Levels of HER2/neu, a Potential Alternative to Tissue Specimen Levels in the Management of Metastatic Breast Cancer: A Case Report and Literature Review," Community Oncology, Vol. 6, No. 8, 2009, pp. 362-364.

[9] R. Iosifidou, G. Galaktidou, A. Ananiadis, N. Bladika, F. Patakiouta and A. Bousoulegas, "VEGF-A, VEGF-C, 
VEGF-R2, EGFR and HER2 in Serum Plus EGFR in Tissue of Patients with Triple-Negative Breast Cancer," Breast Cancer Research, Vol. 11, 2009, p. 13.

[10] Y. Sawada, T. Fujii, H. Takahashi, G. Yokoyama, R. N. Matsubayashi, Y. Inoue, N. Uesugi, S. Momosaki, U. Toh and K. Shirouzu, “A Case of Triple Negative Chest Wall Recurrent Breast Cancer Treated with Capecitabine and Docetaxel Combination Therapy (XT Therapy)," Journal of Cancer Chemotherapy, Vol. 36, No. 5, 2009, pp. 815817.

[11] G. Zhang, M. A. Park, C. Mitchell, H. Hamed, M. Rah- mani, A. P. Martin, D. T. Curiel, A. Yacoub, M. Graf, R. Lee, J. D. Roberts, P. B. Fisher, S. Grant and P. Dent, "Vorinostat and Sorafenib Synergistically Kill Tumor Cells via FLIP Suppression and CD95 Activation," Clinical Cancer Research, Vol. 14, No. 17, 2008, pp. 5385- 5399. doi:10.1158/1078-0432.CCR-08-0469

[12] T. Samuel, W. Fiskus, C. Buser and K. Bhalla, “A Novel Combination Therapy with Histone Deacetylase Inhibitor and Aurora Kinase Inhibitor for 'Triple Negative' Breast Cancers,” Cancer Research, Vol. 69, No. 2, 2009, p. 401. doi:10.1158/0008-5472.SABCS-401 